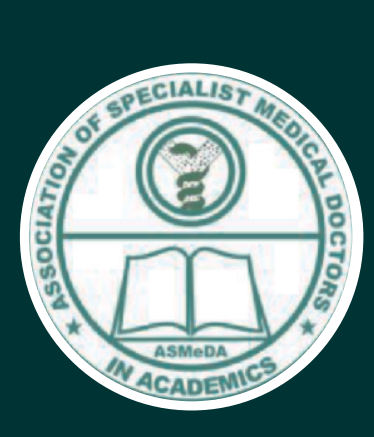

ISSN:Print: 2811-1672

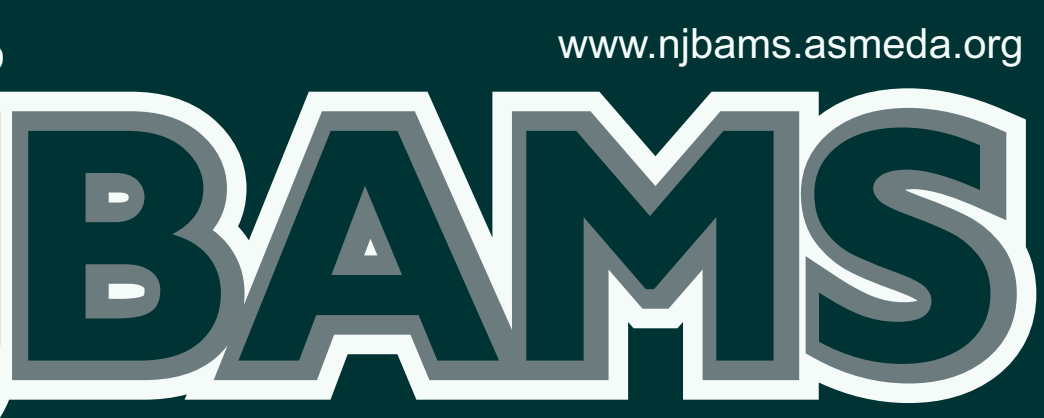

Nigerian Journal of Basic and Applied Medical Sciences

An official publication of the National

ASSOCIATION OF SPECIALIST MEDICAL DOCTORS IN

ACADEMICS (ASMeDA aka MEDSABAMS) 


\title{
Practice of Traditional Uvulectomy and Associated Factors Among Mothers of Under Five Years Children in Sokoto State, Nigeria.
}

\author{
Adamu A ${ }^{1}$, Awosan K.J. ${ }^{2}$, Ango U.M. ${ }^{2}$, Umar M.T. ${ }^{3}$, Bello M.M..${ }^{2}$, Ali M. ${ }^{3}$, Isezuo K.O. ${ }^{1}$, Jiya F.B. ${ }^{1}$, Garba B.I. \\ ${ }^{1}$ Department of Paediatrics, Usmanu Dan Fodiyo University, Sokoto, Nigeria. \\ ${ }^{2}$ Department of Community Health, Usmanu Dan Fodiyo University Teaching, Sokoto, Nigeria. \\ ${ }^{3}$ Department of Pharmacology and Therapeutics, Usmanu Dan Fodiyo University, Sokoto, Nigeria.
}

\begin{abstract}
Introduction: Traditional uvulectomy (TU) is one of the common negative cultural practices in Nigeria, particularly in the Northern Nigeria. The procedure persists in developing countries probably due to low socio-economic status and non-formal level of education. This study aimed to determine the prevalence, reasons, complications and factors associated with the practice of traditional uvulectomy among mothers of under five-year children (0-59 months) in Sokoto state, Nigeria.

Methods: A community based cross sectional study that was conducted in Sokoto State, Nigeria on 320 mothers with their under-five year children from $1^{\text {st }}$ January to $31^{\text {st }}$ March 2021. The study participants were selected using multi stage sampling method. A structured questionnaire was used to collect information and throat examination was done for the children to confirm the practice of TU. Data was analysed using IBM SPSS version 22. The level of significance for Chi square was set at a $p$ value of $<0.05$.

Results: The prevalence of TU is $59.1 \%$ among children under five years of age The major reason for the practice of TU in most $93(29.1 \%)$ of the respondents for their U5 was because of tradition. The main complication after TU was fever $24(35.2 \%)$, followed by bleeding $18(26.5 \%)$. The respondent's educational status $(p=0.004)$ and occupation $(p=0.001)$ were associated with the practice of TU.
\end{abstract}

\section{INTRODUCTION}

Traditional uvulectomy is one of the oldest traditional surgical procedures carried out by traditional surgeons and this practice is as old as mankind. Uvulectomy is the surgical removal of the uvula (1-3). Traditional uvulectomy (TU) is a common practice in areas of the world especially in the Middle East, and Africa (4), it is noted to be one of the common negative cultural practices in Nigeria especially in the North (2), most common among the Hausa ethnic group because of tradition, low socio-economic status and non-formal educational level (2-5). In Sub-Saharan Africa, the practice of TU is very common among children and the benefits perceived to be derived from the belief that uvula leads to various childhood diseases (3).

Traditional uvulectomy in Africa varies in detail, and the practice could either be for ritual or therapeutic purposes (6), in addition, in some communities hymenectomy is as well performed for the children $(6,7)$. The ritual uvulectomy is routinely performed at birth or on the day of naming ceremony (8), while therapeutic uvulectomy is done as a remedy for various ailments (3). No anaesthesia is used during the procedure (9) and haemostasis is achieved with herbal medicine $(2,10)$, The instruments used for the procedure are not sterilised and can lead to complications such as haemorrhage, anaemia, septicaemia, tetanus, risk of Human Immunodeficiency Virus (HIV) infection, hepatitis, neck infection and death $(2,9,10,11)$. Anaemia is usually from postuvulectomy bleeding and sepsis from infections (12). Traditional uvulectomy has been shown to impact negatively on the weight of children that had the procedure $(2,13)$. Aspiration of cut uvula into the lungs and chest infection $(4,10)$, clavicular fracture and obstructive sleep apnoea with snoring from palatal stenosis (1), as

Cite this article as: Adamu A, Awosan K.J., Ango U.M., Umar M.T., Bello M.M., Ali M., Isezuo K.O., Jiya F.B., Garba B.L.Practice of traditional uvulectomy and associated factors among mothers of under five years children in Sokoto state, Nigeria. Nig. J. Basic \& Applied Med. Sci. 2021;1(1);34-39.
Conclusion: Practice of traditional uvulectomy is high and is odue to traditional beliefs. Fever was the most common complication, educational status and occupation were associated with the practice.

Keywords: Mothers, Traditional Uvulectomy, Under five years, Practice, Sokoto Corresponding Author:

Name: Asma'u Adamu,

Address: Department of Paediatrics,

Usmanu DanFodiyo University, Sokoto,Nigeria

Email address: nanadamu@gmail.com well as problems of speech (rhinolalia) (14), haematemesis and malena were other complications of TU (15).

In a cross-sectional study that was done among mothers of under five years children in Jigawa state, Nigeria reported that $90 \%$ of the children had experienced traditional uvulectomy, the reason for the practice was cultural in majority $(77.8 \%)$ of the respondents (2). Similarly, in another study that was conducted in Tanzania among mothers of under five children, showed that $34 \%$ of the children had traditional uvulectomy, the major reason for TU were recurrent sore throat in $33 \%$, cough $(33 \%)$ and vomiting (33\%) (14).

There is paucity of data on the burden of traditional uvulectomy with limited data from North Western Nigeria, the study was therefore carried out to determine the prevalence, reasons, complications and factors associated with the practice of traditional uvulectomy among mothers of under five-years children (0-59 months) in Sokoto state, Nigeria.

\section{MATERIALS AND METHODS}

The study was conducted in Sokoto state, which is one of the six states of the North-western zone of the country. The state is divided into 3 senatorial zones, namely: North, Central and East. It has a total of 23 Local government Areas (LGAs), 5 of which are urban and 18 are rural and a total of 244 political wards. The study was carried out in two selected urban LGAs and two selected rural LGAs. There is a total of 3,666,999 million people in Sokoto based on 2006 census (15), with a projected population of $5,675,488$ people in 2019 , the number of women of child bearing age is $1,204,397$ and total number children under five years is $1,135,098$ (15).

This was a cross sectional study carried out from January to $31^{\text {st }}$ March 2021. The study population comprised of mothers and their children aged 0 to 5 years in Sokoto state. Mothers and their children aged 0 to 5 years who reside in the study area for at least 
1 year were considered eligible for enrolment into the study. Under five years' children who have active Upper Respiratory Tract Infection at the time of the study and those who had previous history of throat surgery in the hospital.

The minimum sample size was calculated to be 291 using Fisher's formula for descriptive studies (16), but 320 study participants were recruited for the study, an $18.7 \%$ prevalence regarding practice of TU from a previous study was used (17), a precision level of $5 \%$, and a response rate of $80 \%$ was anticipated. The eligible study participants were selected using multistage sampling technique. A semi-structured interviewer- administered questionnaire was use to obtain information on socio-demographic characteristics of the respondents and practice of $\mathrm{TU}$ in the respondent's children. Data sheet was used to record information on age, sex, throat examination findings of the eligible under five years' children. The research instruments were pre-tested and five research assistants assisted in questionnaire administration. Throat examination was done by the researcher for presence, absence or amputated uvula.

Completed forms were downloaded from Open Data Kit server in $\mathrm{IBM}^{\circledR}$ SPSS version 22. Age of the respondents and their children were expressed as means and standard deviation, while categorical variables like gender, tribe, religion and age groups were expressed as proportions. Chi-square or where necessary, Fisher's exact test was used to test for association. A p-value of $<0.05$ was considered statistically significant.

Ethical approval for this study was obtained from Sokoto State Ministry of Health Ethical Committee. Written informed consent was also obtained from the study participants.

\section{RESULTS}

In this study a total of 320 mothers who had children less than five years old were interviewed with a response rate of $100 \%$. The mean age of the mothers was $29.9 \pm 8.3 y e a r s$, there ages ranged from 20 to 49 years. The largest proportion of the respondents $136(42.5 \%)$ were in the 25-34years age group, while respondents in the age group 45years and above constituted the lowest percentage $23(7.2 \%)$. Majority of the respondents $315(98.4 \%)$ were Muslims and Hausa's 304(95.0\%). Most of the respondents $140(43.8 \%)$ had Quranic education, only $9(2.8 \%)$ had no any form of education. Housewives 234(73.1\%) constitutes the highest occupation of the respondents. More than half of the respondents $244(76.2 \%)$ have five number of children and below, while $76(23.8 \%)$ have more than five children (Table 1). The respondents husbands are educated up to tertiary level $(37.5 \%)$ and are selfemployed $(55.9 \%)$ (Table 2).

The mean age of the respondent's youngest children was $22.5 \pm 17.7$ months and largest proportion $146(45.3 \%)$ were in the age range 1-12 months. Of the respondent's youngest children, there were $199(62.2 \%)$ males and $121(37.8 \%)$ females; male to female ratio was 1.6:1 (Table 3). Up to $189(59.1 \%$ ) of the under five years' children of the respondents had TU, 176(55.0\%) of the mother's youngest children had TU. About 93(29.1\%) of the respondents practice TU for their U5 because of tradition (Table 4).

Of the 189 children of respondents that had traditional uvulectomy, in $183(96.8 \%)$ of the children it was done before one (figure 1). The most common reason given by respondents for not practicing TU for their under-five years' children was, TU is not necessary $54(41.2 \%)$, while the remaining respondents mentioned fear of complication 33(25.2\%), (Figure 2). In majority 140(79.5\%) of the respondent's children youngest children, permission for TU month of age, while in minority of the practice $3(1.6 \%)$ each was done at 1-5 months and greater than 5 months of age respectively

Table 1: Sociodemographic characteristics of the respondents

\begin{tabular}{|c|c|c|}
\hline Variables & Frequencyl $(=320)$ & Percent $(\%)$ \\
\hline \multicolumn{3}{|c|}{ Mothers'ag(gears) } \\
\hline $15-24$ & 90 & 28.1 \\
\hline $25-34$ & 136 & 42.5 \\
\hline $35-44$ & 71 & 22.2 \\
\hline$\geq 45$ & 23 & 7.2 \\
\hline \multicolumn{3}{|l|}{ Tribe } \\
\hline Hausa/Fulani & 304 & 95.0 \\
\hline Yoruba & 5 & 1.6 \\
\hline Igbo & 1 & 0.3 \\
\hline Others & 10 & 3.1 \\
\hline \multicolumn{3}{|l|}{ Religion } \\
\hline Islam & 315 & 98.4 \\
\hline Christianity & 5 & 1.6 \\
\hline \multicolumn{3}{|c|}{ Educational level } \\
\hline None & 9 & 2.8 \\
\hline Qur'anic only & 140 & 43.8 \\
\hline Primary & 26 & 8.1 \\
\hline Secondary & 75 & 23.4 \\
\hline Tertiary & 70 & 21.9 \\
\hline \multicolumn{3}{|c|}{ Occupational status } \\
\hline House wives & 234 & 73.1 \\
\hline Civil Servant & 62 & 19.4 \\
\hline Self Employed & 11 & 3.4 \\
\hline Unemployed & 7 & 2.2 \\
\hline Student & 6 & 1.9 \\
\hline \multicolumn{3}{|l|}{ Parity } \\
\hline $1-5$ & 244 & 76.2 \\
\hline$>5$ & 76 & 23.8 \\
\hline
\end{tabular}

was given by the father, $13(7.4 \%)$ by both parents, $12(6.8 \%)$ by the mothers and a few by the mother in-law 7(4.0\%) and father-in-law in $4(2.3 \%)$ (Figure 3$)$.

Of the 189 children that had TU, 68(36.0) of the children had complications, the main complication was fever $24(35.2 \%)$, followed

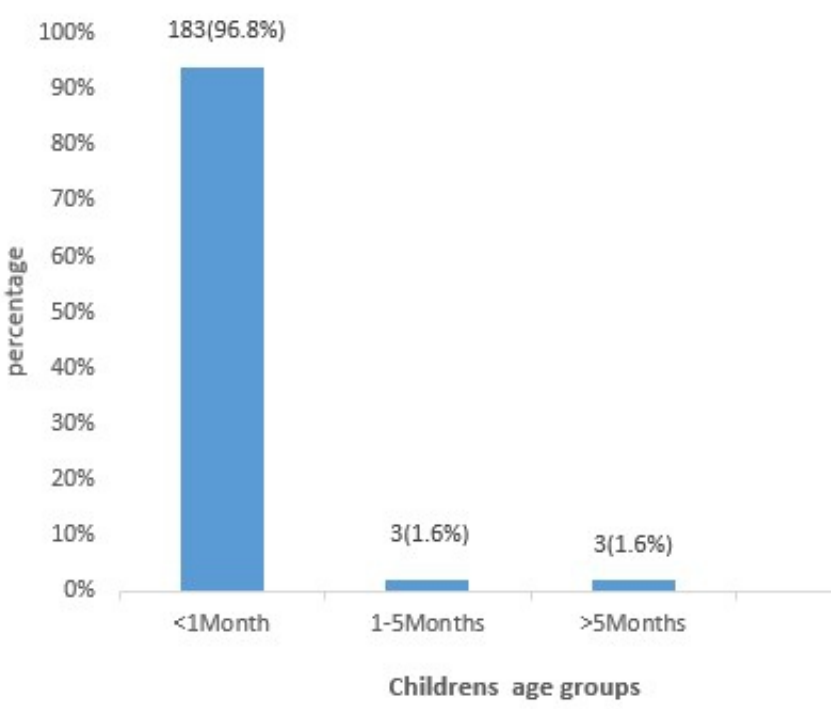

Figure 1: Age of respondents' children at uvulectomy 
by bleeding $18(26.5 \%)$, Failure to Thrive $16(23.5 \%)$, inability to swallow $9(13.2 \%)$ and death in $1(1.5 \%)$ (Table 5). On throat examination of the respondent's youngest children, the uvula was present in 144(45.0\%), amputated in 13(4.1\%) and removed in $163(50.9 \%$ ) (Figure 4). The respondent's educational status, religion, and occupation were associated with practice of TU in this study. Whereas there was no association between age, tribe of the respondents, sex of the respondent's children with the practice of TU (6).

Table 2: Educational level and occupation of the respondents' husbands

Variables $\quad$ Frequency $(n=320) \quad$ Percent $(\%)$

\begin{tabular}{lll}
\hline Educational level & 7 & 2.2 \\
None & 108 & 33.8 \\
Qur'anic & 12 & 3.8 \\
Primary & 73 & 22.8 \\
Secondary & 120 & 37.5 \\
Tertiary & 121 & \\
Occupation & 179 & 37.8 \\
Civil servant & 4 & 55.9 \\
Self employed & 15 & 1.3 \\
Private Sector & 1 & 4.7 \\
Unemployed & & 0.3 \\
Student &
\end{tabular}

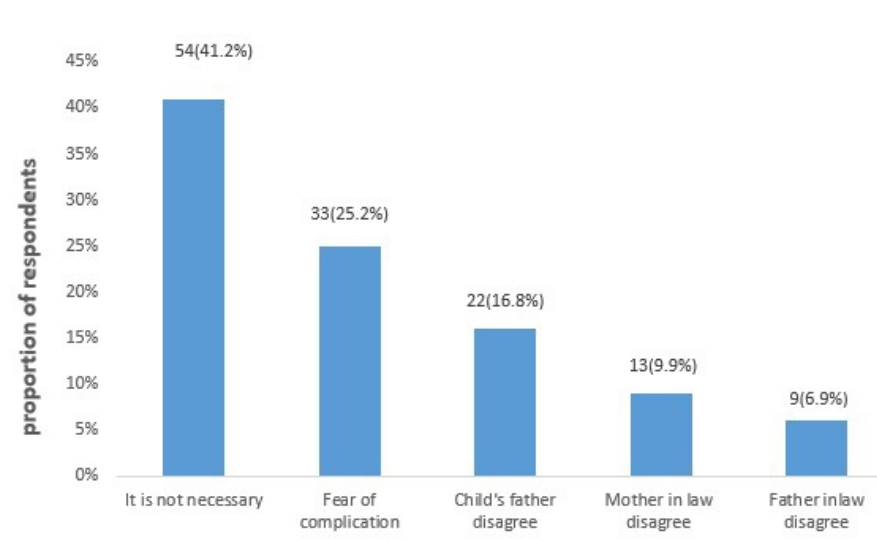

Respondents reasons for not practicing traditional uvulectomy

Figure 2: Respondents reasons for not practicing Traditional Uvulectomy for U5

\section{DISCUSSION}

Socio Demographic findings in this study are similar to the findings of Alebachew et al (18) and Alene and Edris (19) that reported that the largest proportion of their respondents were in the age range 20-34 years. Findings on educational level and occupation are in keeping with the findings of Gebrekirstos et al (20) that reported most of the respondents were jobless and majority $(50.4 \%)$ had primary school level of education, this finding is also in line with the findings of Mboneko et al in Tanzania (14) that reported that most of the parents/guardian had no formal but in contrast to the findings Alebachew et al in Ethiopia that reported more than half of the mothers were civil servant and only a few were unable to read and write (18).

Religious and tribal affiliation in this study is similar to the findings of Prual et al (8) that showed that most of those that had uvulectomy
Table 3: Age and sex of the respondents' youngest child

\begin{tabular}{lll}
\hline Variables & Frequen cy=(320) & Percent (\%) \\
\hline Age (in months) & & \\
$<1$ & 12 & 3.8 \\
$1-12$ & 146 & 45.3 \\
$12-24$ & 63 & 19.7 \\
$35-44$ & 40 & 12.5 \\
$\geq 45$ & 60 & 18.8 \\
Sex & & \\
Male & 199 & 62.2 \\
Female & 121 & 37.8 \\
\hline
\end{tabular}

belong to the Hausa ethnic group. This may be because in some Hausa sub-groups TU is performed routinely as a tradition on the 7th day after birth during naming ceremony to prevent death from swelling of the uvula $(5,6,8)$. This may also be explained by the fact that the inhabitant of the studied location were majority Muslims and Hausa's. On the other hand, Alene and Edris (19), Linto et $\mathrm{al}^{7}$ and Gebrekirstos (20) reports showed that majority of the respondents were Christians which may be explained by the fact that majority of the inhabitants of their studied population were Christians. Respondents number of children in this study is in contrast to the finding of Linto et al and a study done in Ethiopia that reported most of the mothers had a child $(7,18)$. Respondents' husbands level of education in the present study is consistent with the finding of Alebachew et al (18) that showed that most of the respondent's husbands had tertiary level of education and only a few were unable to read and write.

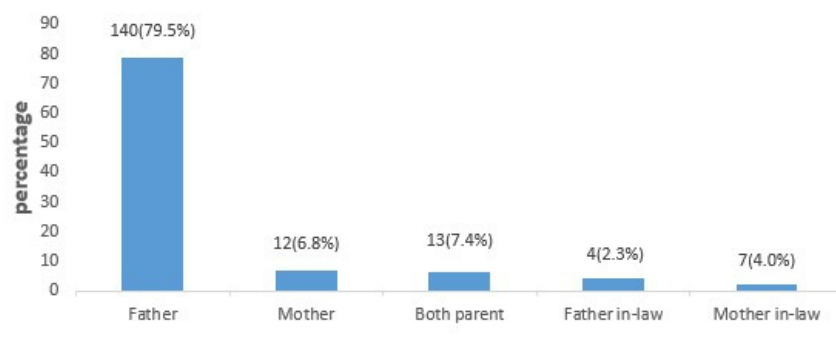

Persons that gave permission for the practice of traditional uvulectomy

Figure 3: Persons that gave permission for practicing Traditional Uvulectomy for U5

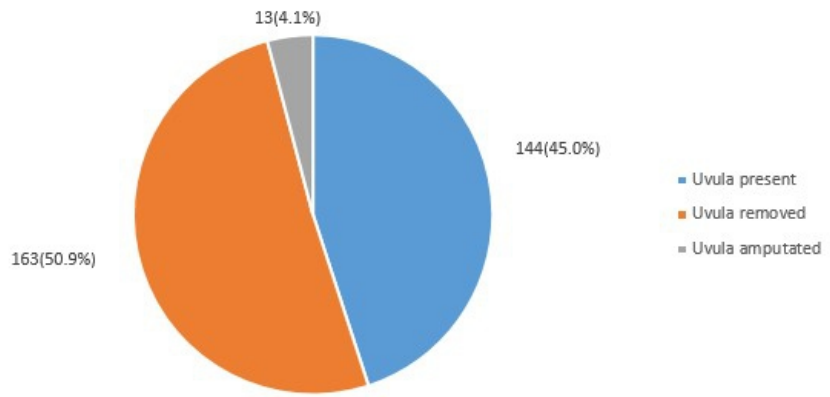

Figure 4: Throat examination findings of the respondent's children

The mean age of the children in this study is similar to the finding of Ajibade et al (2) in Jigawa state, Nigeria which showed that the majority of the respondent's children were within the age bracket 0-12 months and another report from Ethiopia (20) which showed the mean age of the respondents children were $26.28 \pm 15.98$ months and most of the children were in the age range 35-59 months, 
Table 4: Practice and reasons of traditional uvulectomy for under-fives among respondents

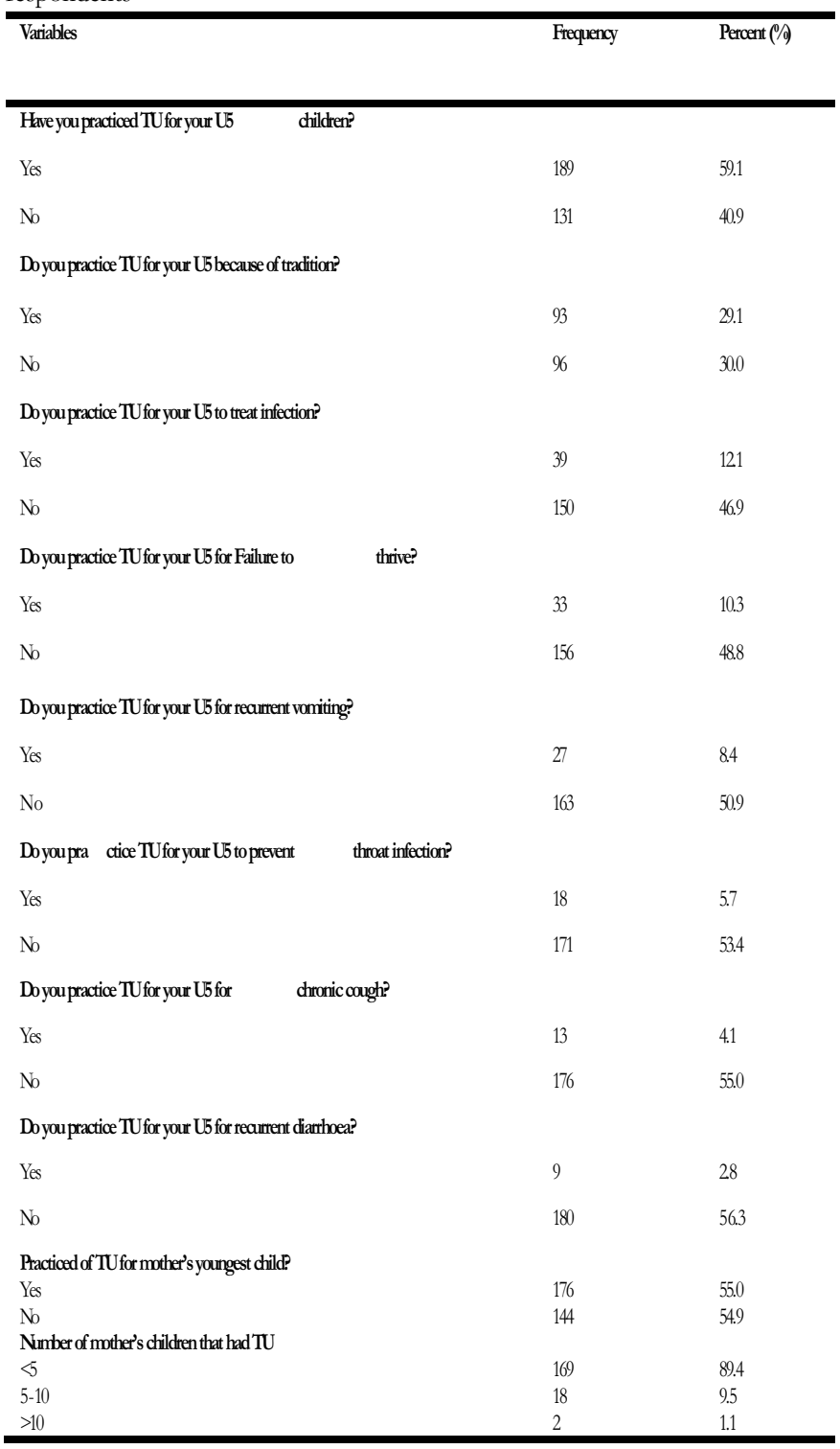

but in contrast to the finding of a study done by Isa et al at University of Maiduguri Teaching Hospital, Borno state, Nigeria which showed that the mean age of the children was $6.7 \pm 4.5$ years and the largest proportion were in the age range 6-10 years (3). This discrepancy may be due to their studied population which were in the age range 15 years and below and also study setting. There were more male children in this study this is in agreement with the findings of a study done at the University of Maiduguri Teaching Hospital, Borno state, Nigeria (3), Jigawa state, Nigeria (2), Sawe et al (21) and Abdullahi et al (5) reported similar finding, but it is in contrast to the findings of Alebachew et al in Ethiopia (18). The prevalence of the under five years' children of the respondents that had TU in this study is lower than the $90.0 \%$ prevalence reported in Northern Nigeria (2) and the $86.9 \%$ reported in Ethiopia (11). On the other hand, the prevalence of TU reported in this study is higher than $18.7 \%$ prevalence obtained among admitted patients in Congo (20), 30.7\% reported from Eritrea (7), 34\% from Tanzania (14) and that of a hospital-based study done in Ethiopia (19). These discrepancies may be due to difference in study setting, study design, period and target population.

The throat examination findings of the respondent's youngest children, is similar to Abdullahi et $\mathrm{al}^{5}$ who made similar observa- tions in which $38.1 \%$ of the subjects had complete removal of the uvula, while $19.0 \%$ had partial removal.

Age at which TU was done in this study is in tandem with an earlier study by Abdullahi et al (5) that reported that for most of the children that had TU in their study, it was done within the first 7 days of life and were in the age range 1-21 days of life, similarly, Alebachew et al (18) reported that most of the TU in their study was performed before one week of life, this gave the impression that tradition is probably an important factor for this practice, also it reinforces the fact that in most cases TU are done as a tradition for preventive measures (2), while in a few of the cases in this study, it was done at 1-5 months $(1.6 \%)$ and greater than 5 months $(1.6 \%)$ of age respectively, while another study reported that most $(68.8 \%)$ of the children had TU at an age range of $0-12$ months (2). Kambale et al (17) reported that the median age at uvulectomy was 11 months, Mboneko and Fabian (14) reported that $68.4 \%$ of the children in their study had TU at two years of age. In another hospital-based study done in Ethiopia, TU was mostly performed between the age of 15-45 days. ${ }^{7}$ Children are vulnerable to TU (5), this may be due to the fact that children are powerless and dependent on adults. Children are usually very vulnerable to such abuses by the traditional healers since consent is only obtained from the parents who believe in the benefits derived from traditional uvulectomy (3).

In this study, reasons for TU agrees with the findings of other studies that reported cultural reasons in most (77.8\%) of the respondents and prevention of sore throat. Similarly, in a previous study done in Eritrea the commonest reasons for $\mathrm{TU}$ was as a result of culture $(60.3 \%)$, followed by throat problems, to prevent respiratory infections, prevent death, to relieve vomiting and to relieve swallowing and feeding problems, another study reported prevention of sore throat $(57.1 \%)$ as the commonest problem, followed by family tradition $(23.8 \%)$ and cough in $9,6 \%$ of the respondents (5), similarly, report from Ethiopia showed that the commonest reason for TU was no modern medicine(79.0\%), fear of recurrence $(31.2 \%)$ and cultural habit $(25.3 \%)$ (18). However, the findings of this study differs with the findings of a previous study by Mboneko et al (14) that reported the main reason for TU in their study in most of the children was cough (32.8\%), followed by recurrent sore throat $(33.2 \%)$, other reasons were tonsillitis $(1 \%)$ and only $0.3 \%$ were due to cultural reasons and the findings of Kambale et al that reported the main reason for TU in their study was fever $(50.0 \%)$, vomiting $(15.8 \%)$ and refusal to suck in $12.4 \%$ (17). The main reason mentioned by respondents for TU in in Axum in Ethiopia study was to prevent swelling, pus and rupture of the uvula which can lead to child death in $68.5 \%$ of the respondents, other reasons reported from their study were no

Table 5: Complication of Traditional uvulectomy

\begin{tabular}{lll} 
Table 5: Complication of Traditional uvulectomy & Percentage (\%) \\
\hline Variables & Frequency & \\
\hline $\begin{array}{l}\text { Ever had complication after } \\
\text { TU(n=189) } \\
\text { Yes }\end{array}$ & 68 & 36.0 \\
No & 121 & 64.0 \\
Type of complication (n=189) & & \\
Fever & 24 & 35.2 \\
Bleeding & 18 & 26.5 \\
Failure to Thrive & 16 & 23.5 \\
Inability to swallow & 9 & 13.2 \\
Death & 1 & 1.5 \\
\hline
\end{tabular}


better medical cure $(12.9 \%)$ and to prevent sore throat by $12.8 \%$ of the respondents (20). From the finding of this study and some other reports $(2,5,7,18)$ tradition is one of the main reason for subjecting children to TU, this is because older adults mostly inherit this age long strong culture and belief that is passed to the young ones from generation to generation, together with lifelong experience $(22,23)$.

Of the 189 children that had TU in this study, $36.0 \%$ of the children had complications, the main complications were fever in $35.2 \%$, followed by bleeding $(26.5 \%)$, FTT $(23.5 \%)$, inability to swallow $(13.2 \%)$ and death $(1.5 \%)$. These findings are consistent with the finding of Linto et al. $^{7}$ that reported $29.4 \%$ of the children developed complications after TU. This is also similar to the findings of Mboneko and Fabian (14) that reported severe bleeding (50\%) and difficulty in swallowing $(50 \%)$ as the commonest complications, mortality was not reported. likewise, Ajibade et al (2) reported bleeding as the most prevalent (88.8\%) complication, followed by infection $(60.0 \%), 67.5 \%$ of the children in their report experienced fever, breathing difficulty in $55.0 \%, 62.0 \%$ of the children developed speech problem and $47.5 \%$ lost weight. In our study none of the children developed speech problem or difficulty in breathing after TU. In line with the present study that showed that the most common complication was fever, Alebachew et al (18) reported a similar finding, followed by anaemia, neck swelling and oropharyngeal injury. The fever in this study may be due to sepsis which might arise from use of unsterilized instruments and the use of these instruments on several children leading to infection $(2,18)$.

The mortality rate was $1.5 \%$ in this study, a higher mortality rate $(11.9 \%)$ following TU was reported by Kambale et al (17) and the factors associated with mortality in their study were HIV infection and acute malnutrition which were not assessed in this study; it was equally discovered by Abdullahi et al (5) in the same study location where the mortality rate following TU was $9.5 \%$ among 21 neonates hospitalised presenting as emergencies, the mortalities in their study was attributed to septicaemia, the mortality in their study may be higher due to the fact that this present study is a community based study restricted to only residents of the state, unlike the Abdullahi et al (5) study been a hospital based study the patients might have come from neighbouring states and countries with complications following TU, It was equally discovered in the Ajibade et al (2) study that the mortality rate was $11.3 \%$ following $\mathrm{TU}$, this is higher than what was found in this study. This is not surprising because the commonest complication in their study was bleeding in most of the children which may explain the high mortality, unlike in our study in which the commonest complication was fever followed by bleeding. Similarly, Olaosun et al (10) reported mortality in a girl with leukemia that had TU. Sawe et al (21) reported a mortality of $21 \%$ among children presenting to the emergency department of Dares Salam, Tanzania.

In this study, the reasons for practicing TU agrees with the findings of other studies that reported cultural reasons in most $(77.8 \%)$ of the respondents and prevention of sore throat $(22.2 \%)$ (2). Similarly, in a previous study done in Eritrea the commonest reasons for TU was as a result of culture $(60.3 \%)$, followed by throat problems $(51.0 \%)$, to prevent respiratory infections $(3.0 \%)$, prevent death, to relieve vomiting $(30.7 \%)$ and to relieve swallowing and feeding problems in $7.3 \%$ of the respondents (7). Another study reported prevention of sore throat $(57.1 \%)$ as the commonest reason, followed by family tradition $(23.8 \%)$ and cough in $9.6 \%$ of the respondents (5). Similarly, report from Ethiopia showed that the commonest reasons for TU were absence of modern medicine $(79.0 \%)$, fear of recurrence $(31.2 \%)$ and cultural habit $(25.3 \%)(20)$. However, the findings of this study differs from the findings of a previous study by Mboneko and Fabian (15) that reported that the main reason for TU in their study in most of the children was cough $(32.8 \%)$, followed by recurrent sore throat $(33.2 \%)$; other reasons were tonsillitis $((1 \%)$ and only $0.3 \%$ were due to cultural reasons. Kambale et al (17) reported that the main reason for TU in their study was fever $(50.0 \%)$, vomiting $(15.8 \%)$ and refusal to suck in $12.4 \%$. The main reason mentioned by respondents for TU in a study done in Axum, Ethiopia were to prevent swelling, pus and rupture of the uvula which could lead to child death in $68.5 \%$ of the respondents, other reasons reported from their study were no better cure in modern medicine $(12.9 \%)$ and to prevent sore throat by $12.8 \%$ of the respondents (20). The finding of this study and some other reports $(2,5,7,18)$ showed that tradition is one of the main reason for subjecting children to $\mathrm{TU}$, this is because older adults mostly inherits this age long strong culture and beliefs that is passed to the young ones from generation to generation, together with lifelong experience $(22,23)$.

In this study, in majority $(79.5 \%)$ of the respondent's youngest children, permission for TU was given by the fathers only without the influence of others, $7.4 \%$ by both parents, $6.8 \%$ by the mothers, $4.0 \%$ by the motherin-laws and a few $(2.3 \%)$ by the fatherin -laws, this is in contrast to the finding of Ajibade et $\mathrm{al}^{2}$ that reported that majority of the children that had TU in their study were taken to the place of uvulectomy by the motherin-laws $(61 \%), 17.5 \%$ by the mothers and only $6.3 \%$ by the fathers, the $f$ inding of this present study is also in 
contrast to the finding of the study by Linto et al, ${ }^{7}$ that reported that most $(80.5 \%)$ of the mothers supported the practice of TU for their children, in-laws $(6.0 \%)$ and only $4.5 \%$ of the husbands supported TU for their children. The role of extended family cannot be downplayed, the finding of this study further supports the finding that women have limited autonomy to make decision concerning their children health and, in most cases, permission to seek health care is given by the mother in-law or husband (24).

The respondent's age was not associated with the practice of TU, this is in consonance with a report from Ethiopia (18) but it is in contrast to the finding of a report from Eritrea (7) which showed no association between the practice of $\mathrm{TU}$ and the age of respondents. The respondent's educational status was associated with the practice of $\mathrm{TU}$, this is in agreement with the findings of previous studies $(3,17,25)$, but in contrast to the findings of Mboneko and Fabian (14), Alene and Edris (19), and Olaosun et al. (10) The finding of this study is in support of the finding of a study in Nigeria by Isa et al (3) which showed significant correlation between reasons for performing TU and educational level of the parents, similarly: Zamir et al (25) and linto et al reported an association between the level of education of the parents/guardian and the practice of TU. It has also been reported that higher levels of educational attainment have been shown to modify knowledge and behaviour regarding health issues (26). The occupation of the respondents was associated with the practice of TU. This agrees with the findings of a study done in Axum town in Ethiopia (20) that reported that harmful traditional practices are more common among jobless mothers, Isa et al (3) reported similar finding. Poverty has been shown to reduce odds of seeking modern medical care and increase the odds of seeking traditional medical care (26).

There was no association between the sex of the respondent's children and the practice of TU, this is not compatible with the finding of a study in Ethiopia (18). Even though there is no association between the sex of respondent's children and practice of TU, the reason for subjecting more male children to uvulectomy is because in some communities' male sex is preferred and considered a pride to the family and community, thus immediate traditional care is given if there is any perceived sign of illness (18). In this current study no association was observed with tribe and the practice of TU. Similarly, Mboneko and Fabian (14) also reported similar findings; this may be because TU is a common practice among some tribes in Nigeria especially in the Northern part of the country and it is usually associated with traditional beliefs and local customs $(23,25)$.

\section{CONCLUSIONS}

Traditional uvulectomy is still an ongoing practice in Sokoto state due to traditional beliefs. The prevalence of traditional uvulectomy in Sokoto is high. The practice is associated with non-formal education in the mother.

\section{RECOMMENDATIONS}

Health education programmes by the government on the dangers of traditional uvulectomy and policies that will empower women like girl child education will help in eradicating the practice of traditional uvulectomy. Health care providers should advocate for legal government intervention to eliminate this harmful traditional practice.

\section{CONFLICT OF INTEREST}

The authors declare no conflict of interest

\section{REFERENCES}

1. Ogah SA, Ocheni SE. Traditional uvulectomy in Lokoja, Nigeria and its associated complications. Asian Journal of Pharmacy, Nursing and Med Scie 2014;2(2):479.

2. Ajibade B, Okunlade J, Kolade O. Harmful Cultural practices: Parents Perceived Effects of Traditional Uvulectomy on under-five Children in Jigawa State, Nigeria. J Dent Med Scie 2013;9(5):8-13.

3. Isa A, Omotara B, Sandabe M, Garandawa H. Parental reasons and perception of traditional uvulectomy in children. Sahel Med J 2011;14(4):210-6.

4. Ravesloot M, De Vries N. A good Shepherd but with obstructive sleep apnoea syndrome: traditional uvulectomy case seiies and literature review J laryngology and otology 2011;1(5).

5. Abdullahi M, Amutta SB. Traditional uvulectomy among neonates: experience in a Nigerian tertiary health instituition. Borno Med J 2016;13(1).

6. Hunter L. Uvulectomy--the making of a ritual. S Afr Med J 1995;85(9):901-2.

7. Linto M, Tekle M, Teklit F, Gebremedhin H, Beraki K, Tekle O, et al. Assesment of the knowledge, Attitude and Practice of child bearing age mothers towards traditional uvulectomy at selected Health facilities of Eritrea in 2015 Int J Med and Health Profession Research. 2015;2(2):51-73.

8. Prual A, Gamatie Y, Djakounda M, Huguet D. Traditional uvulectomy in Niger: a public health problem? Soc Sci Med 1994;39(8):1077-82.

9. Back G, Nadig S, Uppal S, Coatesworth A. Why do we have a uvula? Literature review and a new theory. Clin Otolaryngol. 2004;29:689-93.

10. Olaosun A, Ojemakinde K, Raji A, Adedeji T, Adebola S. Death of a child with Leukaemia subjected to Traditional Uvulectomy. The internet Journal of Third World Medicine. 2006;4(2):1-4.

11. Adoga A, Nimkur T. The Traditionally Amputated Uvula amongst Nigerians: Still an Ongoing Practice J ISRN Otolaryngol 2011;22:4.

12. Abdelhakim E, Abdellah A, Rachid A, Aomar A, Chafiq M. Grisel"s Syndrome: a rare complication following traditional uvulectomy.. Pan Afr Med J 20:62

13. Jeppsson A, Tesfu M, Persson L. Health Care provider's perception on harmful traditional health practices in Ethiopia. Ethiopia J Health Dev 2003 17:35-44.

14. Mboneko K, Fabian F. Traditional uvulectomy and reported complications in under-five children in Mkuranga Distric Pwani Region, Eastern Tanzania. Tanz Dent J 2006;12(2):65-9.

15. UNFPA. Healthcare services in: UNFPA Assisted States of Nigeria, Sokoto state report. Abuja Nigeria: UNFPA Nigeria Country Office A; 2010.

16. Araoye M. Sample size determination in Research methodology with statistics for health and Socl Sci. Ilorin: Nathadex publishers; 2004.

17. Kambale R, Balibuno Y, Isiah F, Masumbako B. Traditional Uvulectomy, a common practice in South Kivu in the Democratic of Congo Med Sante Trop 2018;28(2):176-81.

18. Alebachew B, Minoye B, Yeshabel A. The burden of traditional neonatal uvulectomy among admissions at neonatal intensive care units, North Central Ethiopia, 2019. A triangulated cross sectional study. PLoS ONE. 2019; 15(7).

19. Alene G, Edris M. Knowledge Attitude and practices involved in Harmful Health Behaviour in Demba District,NorthWest Ethiopia Ethiop JHealth 2002;16(2):199-207.

20. Gebrekirstos K, Abebe M, Fantahun A. A cross sectional study on factors associated with harmful traditional practices among children less than 5 years in Axum town, north Ethiopia, 2013. Reprod Health 2014;11(1):46.

21. Sawe H, Mifinanga J, Ringo F, Mwafongo V, Reynoids T, Runyon M. Morbidity and Mortality following Traditional uvulectomy among Children presenting to the Muhimbili National Hospital Emergency Department In Dares Salam , Tanz Emerg Med Intl 2015; 2015:5.

22. Owbingire S, Kamya E, Sohal K. Beliefs about traditional Uvulectomy and Teething : Awareness and perception Among Adults in Tanzanian Rural setting. Ann Int Med Den Res 2018;4(2):25-30.

23. Omoniyi T. Appraisal of harmful traditional practices in Nigeria: Magnitude, justifications and interventions. J social Humanity Edu 2020;1(1):67-78.

24. Dougherty L, Gilroy K, Olayemi A, Ogesanmola O, Ogaga F, Nweze C.. Undestanding factors influencing care seeking for sick children in Ebonyi and Kogi States, Nigeria. BMC Pub Health 2020:20: 746.

25. Olajide T, Gabriel- Alayode O, Olajuyin O, Adeniyi A, Adebgbiji A. Prevalence and Clinical profile of Traditional Uvulectomy in Ekiti, South West, Nigeria. Am J Otolaryngol Head Neck Surg 2020;3(4): 1099.

26. Zamir C, Zamir D, Rishpon D. Traditional practices among children of Ethiopian immigrants in israel. Ambulatory Child Health 1999;5: 351-356.

27. Ahmed S, Goran T, Petzoid M and Kabir Z. Socio-economic status overrides age and gender in determining health seeking behaviour in rural Bangladesh. Bulletin WHO 2005; 83: 109-117. 